\title{
Using General-purpose Sentiment Lexicons for Suicide Risk Assessment in Electronic Health Records: Corpus-Based Analysis
}

André Bittar ${ }^{1}, \mathrm{PhD}$; Sumithra Velupillai ${ }^{1}, \mathrm{PhD}$; Angus Roberts ${ }^{1}, \mathrm{PhD}$; Rina Dutta ${ }^{1,2}, \mathrm{PhD}$

${ }_{1}^{1}$ Institute of Psychiatry, Psychology and Neuroscience, King's College London, London, United Kingdom

${ }^{2}$ South London and Maudsley NHS Foundation Trust, London, United Kingdom

Corresponding Author:

André Bittar, PhD

Institute of Psychiatry, Psychology and Neuroscience

King's College London

16 De Crespigny Park

London, SE5 8AF

United Kingdom

Phone: 44 (0)20 32288553

Email: $\underline{\text { andre.bittar@kcl.ac.uk }}$

\section{Abstract}

Background: Suicide is a serious public health issue, accounting for $1.4 \%$ of all deaths worldwide. Current risk assessment tools are reported as performing little better than chance in predicting suicide. New methods for studying dynamic features in electronic health records (EHRs) are being increasingly explored. One avenue of research involves using sentiment analysis to examine clinicians' subjective judgments when reporting on patients. Several recent studies have used general-purpose sentiment analysis tools to automatically identify negative and positive words within EHRs to test correlations between sentiment extracted from the texts and specific medical outcomes (eg, risk of suicide or in-hospital mortality). However, little attention has been paid to analyzing the specific words identified by general-purpose sentiment lexicons when applied to EHR corpora.

Objective: This study aims to quantitatively and qualitatively evaluate the coverage of six general-purpose sentiment lexicons against a corpus of EHR texts to ascertain the extent to which such lexical resources are fit for use in suicide risk assessment.

Methods: The data for this study were a corpus of 198,451 EHR texts made up of two subcorpora drawn from a 1:4 case-control study comparing clinical notes written over the period leading up to a suicide attempt (cases, $n=2913$ ) with those not preceding such an attempt (controls, $n=14,727$ ). We calculated word frequency distributions within each subcorpus to identify representative keywords for both the case and control subcorpora. We quantified the relative coverage of the 6 lexicons with respect to this list of representative keywords in terms of weighted precision, recall, and $\mathrm{F}$ score.

Results: The six lexicons achieved reasonable precision (0.53-0.68) but very low recall (0.04-0.36). Many of the most representative keywords in the suicide-related (case) subcorpus were not identified by any of the lexicons. The sentiment-bearing status of these keywords for this use case is thus doubtful.

Conclusions: Our findings indicate that these 6 sentiment lexicons are not optimal for use in suicide risk assessment. We propose a set of guidelines for the creation of more suitable lexical resources for distinguishing suicide-related from non-suicide-related EHR texts.

(JMIR Med Inform 2021;9(4):e22397) doi: 10.2196/22397

\section{KEYWORDS}

psychiatry; suicide; suicide, attempted; risk assessment; electronic health records; sentiment analysis; natural language processing; corpus linguistics

\section{Introduction}

\section{Background}

The World Health Organization reports that suicide accounts for $1.4 \%$ of all deaths globally and is the 18th leading cause of death worldwide [1]. Prior history of suicide attempts is the most robust risk factor for completed suicide, and those requiring hospitalization are at the most serious end of the spectrum [2]. However, current methods for assessing a patient's risk of attempting suicide are reported to perform little better 
than chance [3]. Therefore, new methods to understand dynamic features from electronic health records (EHRs) before a hospitalized suicide attempt, distinguishing such periods from clinical narratives at other times, would be of potential clinical utility [4].

EHRs contain structured patient data (eg, age, sex, and ethnicity) and unstructured text that make up the clinical narrative (eg, out-patient letters, event notes from meetings and phone calls with patients or carers, and discharge summaries). Unstructured text is of particular importance in mental health, as much of what is recorded about patients follows face-to-face assessments by clinicians, whose observations and judgments about a patient's experiences and presentation are inevitably influenced by their own training, experience, and implicit biases, and these judgments have a degree of subjectivity when they record this in the clinical narrative [5].

The automatic identification and analysis of subjective judgments in text is known as sentiment analysis [6,7]. This process typically involves the classification of words as expressing either positive or negative polarity, and numerous resources have been developed for this task in nonclinical domains, such as customer reviews [8-11] and social media [12-14]. Research efforts have also focused on the analysis of sentiment within health care-related texts, such as patient feedback forms $[15,16]$, online forums [17], and social networks $[18,19]$.

Recent work has sought to assess the utility of sentiment lexicons for the analysis of subjective judgments in clinical narratives. McCoy et al [20] used a general-domain sentiment analysis tool to extract word polarity features to model the risk of readmission and mortality. The same tool was later used to examine the correlation between word polarity and the risk of suicide attempts [21]. Most recently, Weissman et al [22] carried out a thorough evaluation of six general-domain sentiment analysis tools in predicting the risk of in-hospital mortality of patients in intensive care, tracking the progression of sentiment in clinical notes over time. They concluded that general-domain sentiment tools are not suited to the processing of clinical texts and that domain-specific resources need to be developed. Work in this direction is beginning to emerge [23-25].

These studies have mostly focused on testing the correlation between automatically extracted sentiment values and specific clinical outcomes. However, to our knowledge, there has been no close examination of the terms mapped by general-domain sentiment analysis tools when applied to clinical texts.

\section{Objectives}

Focusing on words with negative and positive polarity, we aimed to determine the coverage of 6 general-purpose sentiment lexicons when applied to a corpus of EHR texts of 2 groups of patients seen by mental health services: (1) patients who had attempted suicide and were hospitalized (cases) and (2) patients with no history of attempted suicide (controls). Adopting methods used in corpus linguistics, we first sought to identify the words that are most representative of the clinical narratives of cases and controls. We then aimed to test the coverage of each sentiment lexicon by comparing these 2 sets of representative words. We sought to ascertain the extent to which these 2 sets of representative words contained general-purpose sentiment words and to what extent these 2 sets contained additional sentiment words not included in the general-purpose lexicons.

\section{Methods}

\section{Corpus Analysis}

\section{Clinical Cohort}

We studied deidentified EHRs of over 250,000 patients from the South London and Maudsley National Health Service Foundation Trust using the Clinical Record Interactive Search (CRIS) database, comprising over 3.5 million text documents [26]. CRIS has been linked with national hospital admission data within a secure safe haven, allowing hospital admission information to be extracted. The deidentified CRIS database has received ethical approval for secondary analysis: Oxford REC C, reference 18/SC/0372. Access is granted upon request to authorized researchers working on projects that have received prior approval from the CRIS Oversight Committee. The data presented in this study can be viewed within the secure system firewall.

Our data set was derived from the EHRs of 17,640 patients. It consisted of 4235 suicide attempt-related (case) admissions and 16,940 nonsuicide attempt-related (control) admissions, sampled according to a 1:4 case-control ratio. Cases were defined as any admission (acute physical or specialist mental health) where there was a suicide attempt (indicated by any of the following codes from the International Classification of Diseases (ICD-10): X6* $\mathrm{X} 7 *$, X80-4*, Y1*, Y2*, Y30-4*, and Y87*) with the admission lasting at least 24 hours. Admissions starting on or after April 1, 2006, and ending before or including March 31, 2017, were considered. Case admissions that had at least one document in the 30 days up to and including the date of the suicide attempt were retained. We also removed admissions with empty documents (text from scanned documents is not always available in CRIS), resulting in a total of 4235 suicide-related admissions. Controls did not have any of the specified ICD-10 codes in the given period, were matched by sex, had to be alive at the admission start date of the corresponding case, and were matched to the same age group (5-year age bands: $<16,16-19,20-24$ to $80-84$, and $>85$ years). Each control also had at least one document in the 30 days up to and including the date of the suicide attempt of the matched case. The controls were chosen to be representative (in terms of age and sex) of the population from which the cases were drawn, and the ratio was based on the epidemiological principle that little statistical power is gained by further increasing the number of controls beyond approximately 4 per case [27]. The key descriptive characteristics of the cohort are presented in Table 1. 
Table 1. Cohort patient- and admission-level statistics.

\begin{tabular}{lll}
\hline Unit of observation & Cases & Controls \\
\hline Patients, $\mathbf{n}(\boldsymbol{\%})$ & $2913(16.51)$ & $14,727(83.49)$ \\
$\quad$ Female & $1730(59.39)$ & $8971(60.92)$ \\
$\quad$ Male & $1183(40.61)$ & $5756(39.08)$ \\
Admissions, $\mathbf{n}(\boldsymbol{\%})$ & $4235(20.00)$ & $16,940(80.00)$ \\
$\quad$ Female & $2598(61.35)$ & $10,392(61.35)$ \\
$\quad$ Male & $1637(38.65)$ & $6548(38.65)$ \\
Age (years), mean (SD) & $34.4(15.3)$ & $34.4(15.4)$ \\
\hline
\end{tabular}

\section{EHR Corpus}

Our corpus comprised all EHR texts for each of the 2 subgroups in our clinical cohort: (1) suicidal case admissions and (2) nonsuicidal controls.

Our use of a 1:4 case-control study design for admissions means we expect a disparity in document number and word count between subcorpora. However, there are only $77.92 \%$ $(55,643 / 71,404)$ more control documents $(n=127,047)$ than case documents $(n=71,404)$, rather than the $300 \%$ difference that might be expected for 1:4 sampling of random patients. Following data preprocessing (refer to the Data Preparation subsection), the mean lexical word count for case documents $(n=117.4)$ is higher than that for control documents $(n=103.9)$, so that the overall word (token) count ratio is not 1:4 but approximately 1:1.6, whereas the mean unique word (type) count ratio is approximately 1.5 . The basic descriptive statistics for the corpus are shown in Table 2. The distribution of documents per patient followed a non-normal distribution, as shown in Multimedia Appendix 1.

Table 2. Electronic health record corpus descriptive statistics.

\begin{tabular}{llll}
\hline Unit of observation & Cases & Controls & Total \\
\hline Word tokens, $\mathrm{n}$ & $8,385,643$ & $13,198,250$ & $21,583,893$ \\
Word types, $\mathrm{n}$ & 109,024 & 162,696 & 206,866 \\
Type-token ratio ${ }^{\mathrm{a}}, \%$ & 1.30 & 1.23 & 0.96 \\
Documents, $\mathrm{n}$ & 71,404 & 127,047 & 198,451 \\
Number of words per document, mean (SD) & $117.4(219.1)$ & $103.9(252.7)$ & $108.8(241.3)$ \\
\hline
\end{tabular}

${ }^{\mathrm{a}}$ Type-token ratio $=$ number of word types $/$ number of word tokens $\times 100$.

\section{Data Preparation}

All texts were preprocessed using the Natural Language Processing (NLP) library spaCy (v2.0.12) [28], applying the following steps: word tokenization, part-of-speech tagging, and lemmatization (to use the base form of words). We removed stop words using the Natural Language ToolKit [29] stop words list for English and lowercased all words for our analyses. All codes were made available on GitHub [30].

\section{Identifying Representative Keywords}

To answer our questions concerning the coverage of each lexicon, we adopted methods based on word frequency distributions, commonly used in corpus linguistics, as described further in Multimedia Appendix 1 (C) [31-34]. We first determined which keywords were most representative of each subcorpus (suicidal case admission texts and nonsuicidal control texts) by calculating the relative word frequency ratios between subcorpora. Following recommendations from previous research in corpus linguistics [31-33] and given the non-normal distribution of documents between patients, we then applied the nonparametric Mann-Whitney $U$ test to determine the statistical significance of word frequency differences (FreqDiff $(w)$ for a given word w) between subcorpora. We only retained words that occurred in both the case and control subcorpora, leaving a total of 64,854 unique token types. Words appearing in only one or other subcorpora were relatively infrequent compared with those that were common to both subcorpora. For example, the most frequent case-only keywords were identifying initials, with a maximum frequency of 20.2 words per million (wpm), whereas the most frequent control-only keywords were persons' names, with a maximum frequency of $34.4 \mathrm{wpm}$.

\section{Sentiment Lexicon Analysis}

\section{Sentiment Lexicons}

We examined six different sentiment lexicons that were developed for nonclinical domains. Various dimensions of sentiment and affect have been studied, including emotion, valence-arousal-dominance, and polarity. We focused solely on lexicons that represent this last aspect, that is, negative and positive sentiment polarity. Along with assigning negative and positive polarity, some sentiment analysis tools also assign a value for words that do not convey semantic polarity (ie, neutral words). However, we only considered words that express positive and negative sentiments, as not all the lexicons in this study contain neutral terms. Therefore, we filtered out any 
neutral words. Furthermore, for the sake of comparison, we only examined binary sentiment values rather than degree scores, which only some lexicons provide. We selected the following lexicons for this study: AFINN [35], the NRC Emotion Lexicon (commonly known as EmoLex) [36], Linguistic Inquiry and Word Count (LIWC) [37], the Opinion lexicon [9], the Pattern lexicon [38], and SentiWordNet [39]. The lexicons differ in terms of the forms they contain (words, lemmas, and regular expressions). We applied each one as-is to the appropriately preprocessed corpus (eg, words or lemmas) to compare them, as they have been used in other studies. We provide details of the lexicons, including preprocessing and filtering, in Multimedia Appendix 1 (B) [9,35-44]. Table 3 summarizes some of the main characteristics of each of these lexicons, including size before (original size) and after (filtered size) filtering out neutral entries.

Table 3. Characteristics of the 6 sentiment lexicons.

\begin{tabular}{|c|c|c|c|c|c|c|}
\hline Lexicon & Source & $\begin{array}{l}\text { Automatic } \\
\text { term selection }\end{array}$ & Intended domain & Term type & $\begin{array}{l}\text { Original size } \\
\text { (entries), } \mathrm{n}\end{array}$ & $\begin{array}{l}\text { Filtered size (number } \\
\text { of entries), n (\%) }\end{array}$ \\
\hline AFINN & Various web-based word lists & No & Microblogs & Word forms & 3478 & $3478(100.00)$ \\
\hline EmoLex & $\begin{array}{l}\text { Macquarie Thesaurus, General } \\
\text { Inquirer, WordNet }\end{array}$ & No & General & Word forms & 14,182 & $5555(39.17)$ \\
\hline LIWC $^{\mathrm{a}}$ & $\begin{array}{l}\text { Various dictionaries and the- } \\
\text { sauruses }\end{array}$ & No & Personal narratives & $\begin{array}{l}\text { Word forms and } \\
\text { regular expres- } \\
\text { sions }\end{array}$ & 1371 & $1371(100.00)$ \\
\hline Opinion & Web crawl of product reviews & Yes & Product reviews & Word forms & 6789 & $6789(100.00)$ \\
\hline Pattern & Subset of WordNet & No & Product reviews & Lemmas+POS ${ }^{b}$ & 2896 & $2293(79.18)$ \\
\hline SentiWordNet & WordNet & Yes & General & $\begin{array}{l}\text { Synset Lem- } \\
\text { mas+POS }\end{array}$ & 117,659 & $39,746(33.78)$ \\
\hline
\end{tabular}

${ }^{a}$ LIWC: Linguistic Inquiry and Word Count.

${ }^{b}$ POS: part of speech.

\section{Lexicon Coverage}

We assessed the coverage of each lexicon in three different ways:

1. Global coverage: The percentage of sentiment-bearing lexical entries that appeared in the list of (unique) words for each subcorpus. Further details are provided in Multimedia Appendix 1 (D).

2. Keyword coverage: The proportion of case and control keywords covered by the sentiment-bearing terms of a lexicon. First, we calculated the percentage of keywords identified by each lexicon for each subcorpus. Second, we used metrics common to information retrieval, namely, weighted precision $\left(\mathrm{P}_{\mathrm{w}}\right)$, recall $\left(\mathrm{R}_{\mathrm{w}}\right)$, and $F$ score $\left(\mathrm{F}_{\mathrm{w}}\right)$, which we calculated for each lexicon across the unordered set of all keywords, using word ranking as the weighting. Details of our calculations, including formulae, are provided in Multimedia Appendix 1 (D). A lexicon's precision shows how many case keywords it correctly identifies as a proportion of all the keywords it contains. The inclusion of control keywords in a lexicon, therefore, penalizes precision. In contrast, recall indicates the number of case keywords that the lexicon correctly identifies from the entire list of case keywords. The absence of case keywords from a lexicon results in a penalty on recall. $F$ score provides a combination of the preceding 2 metrics and an overall quantified evaluation of a lexicon's keyword coverage.

3. Sentiment coverage: The sentiment polarity (positive or negative) that lexicons assigned to matched keywords for each subcorpus.

\section{Results}

\section{Corpus Analysis}

The step of generating representative keywords for each subcorpus (refer to the Corpus analysis subsection) resulted in a list of 3382 keywords. Sorted by decreasing the frequency difference, the top words (with FreqDiff $>0$ ) are representative of the suicidal case subcorpus (2360 keywords). Similarly, sorting in ascending order, top words (with FreqDiff<0) are representative of the nonsuicidal control subcorpus (1022 keywords). Table 4 shows the 10 top-ranking keywords for each subcorpus. In this table, we show each word's rank as well as its frequency in the whole corpus, the frequency difference between case and control subcorpora, and the frequency ratio for the word across the subcorpora. We provide a similar list of the top 100 keywords in Multimedia Appendix 2. 
Table 4. Ranked keyword list for suicidal case and nonsuicidal control subcorpora.

\begin{tabular}{|c|c|c|c|c|c|c|c|c|c|}
\hline \multicolumn{5}{|c|}{ Suicidal case keywords } & \multicolumn{5}{|c|}{ Nonsuicidal control keywords } \\
\hline Rank & Word & $\begin{array}{l}\text { Freq }^{\mathrm{a}} \text { (words per } \\
\text { million) }\end{array}$ & Freq $\operatorname{diff}^{\mathrm{b}}$ & Freq ratio ${ }^{c}$ & Rank & Word & $\begin{array}{l}\text { Freq (words per } \\
\text { million) }\end{array}$ & Freq diff & Freq ratio \\
\hline 1 & QQQQQ $^{\mathrm{d}}$ & 9779.1 & 3545.7 & 1.6 & 1 & $\mathrm{ZZZZZ}^{\mathrm{d}}$ & 35657.1 & -3801.4 & 1.1 \\
\hline 2 & self & 4278.5 & 2060.9 & 1.9 & 2 & mental & 3092.5 & -1242.5 & 1.4 \\
\hline 3 & harm & 2916.2 & 1673.4 & 2.4 & 3 & $\mathrm{mr}$ & 1197.9 & -1138.1 & 2.0 \\
\hline 4 & ward & 5554.7 & 1597.1 & 1.4 & 4 & appointment & 1583.5 & -1124.5 & 1.7 \\
\hline 5 & overdose & 1717.0 & 1392.8 & 5.3 & 5 & medication & 3756.5 & -1017.4 & 1.3 \\
\hline 6 & staff & 5670.0 & 1389.4 & 1.3 & 6 & health & 2282.2 & -771.1 & 1.3 \\
\hline 7 & suicidal & 2072.5 & 1256.2 & 2.5 & 7 & please & 1305.9 & -703.6 & 1.5 \\
\hline 8 & said & 5725.4 & 1137.7 & 1.3 & 8 & state & 1640.3 & -694.4 & 1.4 \\
\hline 9 & alcohol & 2276.2 & 1102.4 & 1.9 & 9 & service & 1190.6 & -678.1 & 1.6 \\
\hline 10 & a\&e & 1534.1 & 1089.5 & 3.5 & 10 & road & 729.3 & -596.2 & 1.8 \\
\hline
\end{tabular}

${ }^{\mathrm{a}}$ Freq: word frequency.

${ }^{\mathrm{b}}$ Freq diff: frequency difference.

${ }^{\mathrm{c}}$ Freq ratio: frequency ratio between subcorpora.

${ }^{\mathrm{d}}$ Masking strings created by the electronic health record deidentification process: QQQQQ for relative or close contact identifiers and ZZZZZ for patient identifiers.

For the suicidal case subcorpus, the top keyword "QQQQQ" is a placeholder for anonymized names of relatives or close contacts of the patient created by a bespoke deidentification algorithm used in CRIS [45]. This could indicate concerns of relatives or carers being reported to staff over the patient's status. Other top keywords directly relate to the theme of suicide attempts (overdose, suicidal, and a\&e [accident and emergency]). The frequency ratio indicates that overdose is over 5 times and $a \& e$ is over 3.5 times more frequent in the case subcorpus than in the control subcorpus. Other words relate to hospitalization (ward and staff) and self-harm (self and harm).

Visual inspection shows that self and harm frequently co-occur in noun phrases such as harm to self and self-harm (which was incorrectly segmented into 2 tokens by the tokenizer). Furthermore, harm also occurs with reflexive pronouns, for example, harm himself/herself, also referencing self-harm events. Alcohol is also clinically relevant because both chronic alcohol use disorders and acute use of alcohol confer risk for attempted suicide.

In contrast, for the control subcorpus, the top keyword "ZZZZZ" is a placeholder for anonymized patient identifiers. These top keywords are more generic terms that may be found in most types of clinical notes (eg, mental, health, and state) and some are likely to be derived from correspondence (eg, $m r$, appointment, and please). Although the top control keywords are significantly more frequent than those in the case subcorpus, the frequency difference and ratio are globally less marked than for case keywords. The median absolute frequency difference (FreqDiff) for the top 10 control keywords is 894.2, compared with 1391.1 for cases. The corresponding median frequency ratios (FreqRatio) are 1.90 for cases and 1.45 for controls. This indicates that keywords for suicide-related texts are more strongly representative of the case subcorpus than the keywords for the control subcorpus. This may reflect the fact that cases have a distinct unifying feature of being included for their hospitalized suicide attempt, whereas control admissions were from any period as long as they did not precede a suicide attempt. It should be noted that no suppositions about the sentiment associated with these keywords were made.

\section{Sentiment Lexicon Analysis}

We first assessed the global coverage of sentiment lexicons (refer to Multimedia Appendix 1 (E) for details). The figures for global coverage are summarized in Table 5. 
Table 5. Term type and token counts for each lexicon in case and control subcorpora and whole corpus. Percentages for control words are shown as (raw/adjusted). Figures are in descending order of lexicon (filtered) size.

\begin{tabular}{|c|c|c|c|c|c|c|c|}
\hline \multirow[t]{2}{*}{ Lexicon } & \multirow{2}{*}{$\begin{array}{l}\text { Filtered } \\
\text { size }\end{array}$} & \multicolumn{3}{|l|}{ Word types } & \multicolumn{3}{|l|}{ Word tokens } \\
\hline & & Case, $\mathrm{n}(\%)$ & Control, n (\%) & $\begin{array}{l}\text { Whole corpus, } \\
\mathrm{n}(\%)\end{array}$ & Case, n (\%) & Control, n (\%) & Whole corpus, $\mathrm{n}(\%)$ \\
\hline $\begin{array}{l}\text { SentiWord- } \\
\text { Net }\end{array}$ & 39,746 & $9843(9.02)$ & $\begin{array}{l}12,429 \\
(7.64 / 5.12)\end{array}$ & $13,373(6.46)$ & $4,234,058(50.49)$ & $\begin{array}{l}8,603,932 \\
(65.19 / 41.42)\end{array}$ & $12,837,990(59.48)$ \\
\hline Opinion & 6789 & $3111(2.85)$ & $3662(2.25 / 1.51)$ & $3821(1.85)$ & 979,804 (11.68) & $\begin{array}{l}1,959,007 \\
(14.84 / 9.43)\end{array}$ & $2,938,811(13.62)$ \\
\hline EmoLex & 5555 & $3733(3.42)$ & $4260(2.62 / 1.75)$ & $4426(2.14)$ & $1,456,097(17.36)$ & $\begin{array}{l}2,869,472 \\
(21.74 / 13.81)\end{array}$ & $4,325,569(20.04)$ \\
\hline AFINN & 3478 & $2529(2.32)$ & $2781(1.71 / 1.15)$ & 2845 (1.37) & $1,274,283(15.20)$ & $\begin{array}{l}2,532,261 \\
(19.19 / 12.19)\end{array}$ & 3,806,544 (17.64) \\
\hline Pattern & 2293 & $1101(1.01)$ & $1243(0.76 / 0.51)$ & $1296(0.63)$ & $910,369(10.86)$ & $\begin{array}{l}1,957,386 \\
(14.83 / 9.42)\end{array}$ & $2,867,755(13.29)$ \\
\hline LIWC $^{\mathrm{a}}$ & 1371 & $3708(3.40)$ & $5824(3.58 / 2.40)$ & $6269(3.03)$ & $620,546(7.40)$ & $\begin{array}{l}1,830,216 \\
(13.87 / 8.81)\end{array}$ & 2,450,762 (11.35) \\
\hline
\end{tabular}

${ }^{a}$ LIWC: Linguistic Inquiry and Word Count.

SentiWordNet, by far the largest lexicon, has the widest coverage of approximately $60 \%$ of all tokens (6.46\% types) in the entire corpus. The pattern has the lowest word-type coverage for both subcorpora and the whole corpus (0.63\%). Although LIWC has the fewest lexical entries (1371), its use of regular expressions that capture multiple word forms means it maps more individual word types (but has the lowest coverage of tokens, $11.35 \%$ on the whole corpus). Despite having approximately 1200 and 3300 fewer entries than Opinion, respectively, EmoLex and AFINN both have a substantially higher coverage of word tokens over the larger lexicon. EmoLex also has a slightly higher coverage of token types. This may be a consequence of the manner in which these lexicons were constructed and the sources from which they were derived. We review this issue in the Discussion section.

With the exception of LIWC, all lexicons show higher coverage of word types in the case subcorpus than in the control subcorpus. The same trend was observed when considering the adjusted percentages for word tokens. This suggests that there is generally more sentiment (as defined in these lexicons) expressed in the case subcorpus than in the control subcorpus, assuming an artificial scenario in which there are an equal number of words of each. However, if no adjustment for word frequency disparities across subcorpora is made, the opposite tendency is observed for all lexicons.

This notion of coverage does not take into account the representativeness of the words in question. To capture this crucial characteristic, we examined the proportion of keywords (word types) from each subcorpus containing each lexicon (keyword coverage; refer to the Corpus Analysis subsection and Multimedia Appendix 1 [D]). The overall proportional coverage of keywords is shown in Table 6.

Table 6. Case and control keywords that appear in each sentiment lexicon, in descending order of lexicon (filtered) size. The total number of keywords for the case subcorpus is 2360 and for the control subcorpus is 1022 .

\begin{tabular}{llll}
\hline Lexicon & Filtered size & Case, $\mathrm{n}(\%)$ & Control, $\mathrm{n}(\%)$ \\
\hline SentiWordNet & 39,746 & $604(25.6)$ & $231(22.6)$ \\
Opinion & 6789 & $192(8.1)$ & $60(5)$ \\
EmoLex & 5555 & $277(11.7)$ & $117(11.4)$ \\
AFINN & 3478 & $238(10.1)$ & $74(7)$ \\
Pattern & 2293 & $115(4.9)$ & $39(3)$ \\
LIWC $^{\text {a }}$ & 1371 & $181(7.7)$ & $48(4)$ \\
\hline
\end{tabular}

${ }^{a}$ LIWC: Linguistic Inquiry and Word Count.

As with global coverage, keyword coverage is correlated with lexicon size, with LIWC being the exception. Again, when examining only the most representative words for each subcorpus, Opinion, the second largest resource, has substantially lower coverage than both EmoLex and AFINN, which are smaller in size, the latter resource numbering only half as many keywords among its entries.

Evaluating the lexicons from an information retrieval perspective revealed the extent to which each lexicon strikes a balance 
between the inclusion of case keywords and the exclusion of control keywords, accounting for the representativeness of the words identified. As shown in Table 7, all lexicons provided reasonable weighted precision (0.53-0.72). However, weighted recall and weighted F-score, which varied substantially across lexicons, were very low (0.04-0.36).

Table 7. Weighted metrics for each lexicon in descending order of weighted F score.

\begin{tabular}{llll}
\hline Lexicon & Weighted precision & Weighted recall & Weighted $F$ score \\
\hline SentiWordNet & 0.68 & 0.36 & 0.47 \\
EmoLex & 0.68 & 0.18 & 0.29 \\
AFINN & 0.72 & 0.15 & 0.25 \\
Opinion & 0.68 & 0.11 & 0.18 \\
LIWC & 0.69 & 0.10 & 0.17 \\
Pattern & 0.53 & 0.04 & 0.07 \\
\hline
\end{tabular}

${ }^{\mathrm{a}}$ LIWC: Linguistic Inquiry and Word Count.

These results show that, of all the lexicons we tested, SentiWordNet provides the best balance between precision and recall over keywords from the 2 subcorpora. Owing to its size, it obtained the highest recall. This indicates that it contains more of the most highly ranked case keywords than the other lexical resources. It also achieved precision on par with the other lexicons, indicating that the words it identifies are often high-ranking keywords from the suicide-related case subcorpus. The pattern lexicon achieved significantly lower results in terms of weighted precision and recall than all other lexicons, despite being larger than some of these. This suggests that its included sentiment terms are of a somewhat different nature and do not contribute a clear signal for distinguishing representative case keywords from control keywords.
Overall, as tools for distinguishing suicide-related from nonsuicide-related clinical notes, this evaluation, in particular the recall figures, shows that the most representative keywords in both subcorpora are not sentiment bearing, as defined in all these lexicons, thus indicating that there is a need for further analysis of the representative subcorpus keywords to better understand their characteristics.

Finally, we examined the distribution of sentiment among the top-ranking representative keywords for each subcorpus (sentiment coverage). Figure 1 shows the ranks of the top 100 keywords each lexicon contains for the case and control subcorpora. In addition to plotting the ranks of words featured in each lexicon, we also indicate, through color and shape coding, the polarity associated with each term. 
Figure 1. Comparative sentiment lexicon coverage of top 100 ranked words for the suicidal case and nonsuicidal control subcorpora.

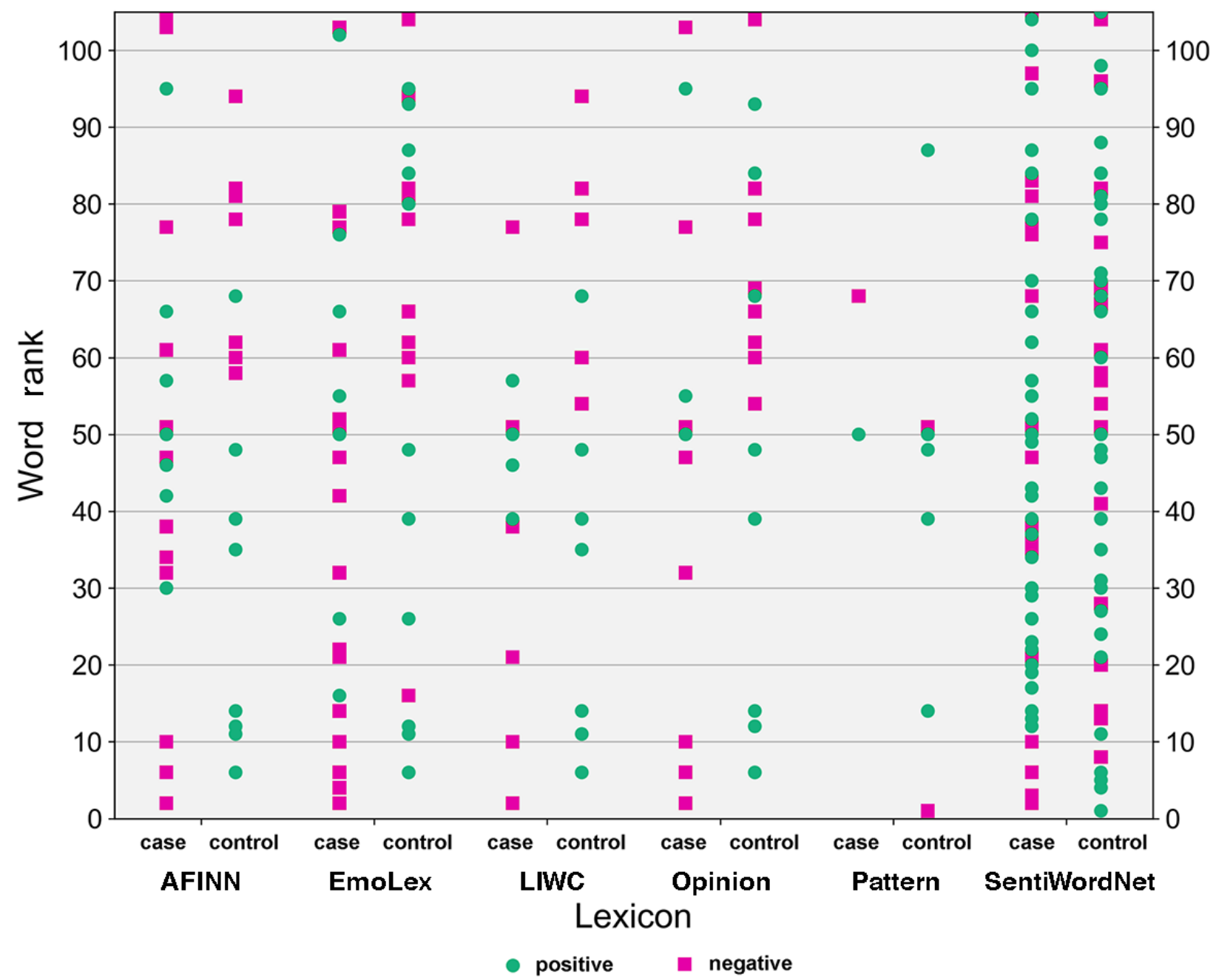

In terms of sentiment coverage, AFINN, EmoLex, LIWC, and Opinion mark a clear distinction between the top case and control keywords. These lexicons assign negative sentiment to high-ranking case keywords (eg, harm [ranked third], risk [11th], kill [52nd], and pain [78th]) and positive sentiment to top control keywords (eg, please [seventh], calm [40th], and pleasant [49th]), and negative also to certain high-ranking control keywords (eg, aggressive [61st], illness [63rd], and anxiety [83rd]).

Only 2 high-ranking keywords for cases appeared in the Pattern lexicon: these were safe [51st], which was the only one of the top 100 ranked words consistently found for cases across all 7 lexicons, and past [68th], which only appeared in Pattern and was ascribed a negative polarity (further discussed in the Discussion section). Calm [40th] and pleasant [49th] were the only top 100 keywords found consistently for controls across all 6 lexicons, and these were ascribed a positive polarity by all except SentiWordNet. This unexpected assignment of sentiment (the adjective calm is given a heavily negative score in SentiWordNet, whereas anxious, borderline, cutting, and concern are positive) highlights the importance of studying the underlying assumptions in off-the-shelf tools and their potential implications when applying them for a new use case.
For SentiWordNet, sentiment of top keywords is mixed, with a higher proportion of positive sentiment keywords in both subcorpora, although it assigned more negative sentiment for controls and for a greater proportion of the high-ranked keywords. This shows that despite having a larger lexical coverage, the sentiment coverage of this lexicon may not be sufficiently consistent to reliably distinguish the 2 populations.

It is important to note that 51 of the top 100 keywords for the case subcorpus were not identified by any of the lexicons. These included self, staff, said, alcohol, and $a \&$, all in the top 10 (Table 4), as well as further highly clinically relevant (although not necessarily sentiment bearing) words such as paracetamol (ranked 25th, FreqDiff=524.6, FreqRatio=4.5), the abbreviation od (used variably in psychiatry to mean either overdose or omne in die [once a day] with respect to medication; ranked 29th, FreqDiff $=498.2$, FreqRatio=2.2), ambulance (ranked 57th, FreqDiff $=340.9$, FreqRatio=3.3), the plural form overdoses (ranked 68th, FreqDiff=314.0, FreqRatio=7.6), and the acronym $d s h$ (deliberate self-harm; ranked 83rd, FreqDiff=275.1, FreqRatio=3.4). The frequency ratio of these words shows that they were many times more frequent in suicide-related case notes than in the control corpus. Over the entire list of case keywords, only $33.35 \%$ (787/2360) were assigned a sentiment value by at least one of the lexicons. Furthermore, 51 of the top 
100 control keywords were also absent from all lexicons, many of which pertain to correspondence (eg, $m r$, appointment, and fax). We refer the reader to Multimedia Appendix 2 for further details.

\section{Discussion}

\section{Implications for Suicide Risk Assessment Lexicon Development}

The list of representative keywords extracted from our corpus shows that the notion of sentiment generally adopted in the field of NLP is not the most appropriate semantic category for identifying terms that typify case notes of suicidal patients. Many of these terms do not carry an obvious negative or positive polarity, as defined in the tested sentiment lexicons.

Our analysis also showed that there is a need for further analysis of the assignment of sentiment polarity by these tools when applied on new use cases.

Furthermore, many of the keywords we identified as representative of suicide-related case notes were neutral with respect to sentiment, which is expected, and representative case keywords extracted in our study indicate that they are distinct from control keywords, but not all such terms would necessarily be sentiment bearing.

Our results show that these sentiment lexicons built using validated lexical resources, such as dictionaries or thesauri (eg, EmoLex), had higher combined precision and recall results than those derived from semiautomatic processes over large open-domain text corpora (eg, Opinion, built by web crawling).

\section{Guidelines for Building Sentiment Lexicons for Suicide} Risk Assessment

Following the work of Deng et al [24], one solution to the unsuitability of general-domain lexical resources for the clinical domain consists of defining the notion of sentiment for the analysis of clinical texts, and in the present case, of mental health (Guideline 1). This could allow the assignment of polarity to terms that do not feature in general-purpose lexical resources. In the case of suicide risk assessment, this might include the assignment of negative polarity to terms such as $a \& e$, overdose, alcohol, $d s h$, and plan, which were not assigned a polarity value by the lexicons we tested.

In light of our results, a suggested strategy for building a suicide risk assessment lexicon may be to use corpus word frequencies as a guide to inclusion of words in a lexical resource that would remain agnostic with respect to sentiment (Guideline 2) and instead labeling terms as trigger or risk factor words (Guideline 3). Such a strategy would avoid the problem of assigning sentiment to words which, although highly representative of suicide-related texts, do not have an obvious sentiment value. This would also obviate the need to assign a polarity to terms that may be ambiguous in the sentiment they express, being either positive or negative depending on context (eg, low [emotion] vs low [risk]), although the more general problem of polysemy remains.
For clinically relevant terms, specialized psychiatric dictionaries or health care terminologies could be beneficial in creating a targeted lexical resource for suicide risk assessment (Guideline 4). For example, certain risk factors for suicide (eg, previous suicide attempts, depression, and substance misuse) and protective factors (eg, effective clinical care, family, and community support) are already well-known clinical features. Therefore, these concepts and associated terms should be reflected in any lexicon aiming to identify periods of increased suicide risk in clinical notes. One caveat that must be kept in mind is that many terms contained in specialized clinical terminologies are not written in EHRs by clinicians [46], meaning that term selection should be carried out by domain experts with a general awareness of typical target corpora.

Automated approaches to extracting terms from large corpora have become common in the field of NLP, including the creation of sentiment lexicons [47-49]. These techniques provide a means to increase the coverage of relevant terms, although it is preferable to implement some mechanism to ensure that the criterion of relevance is respected. Incorporating a domain-specific corpus-based notion of term representativeness into automatic lexicon induction procedures [50] is one way of refining term selection, filtering out terms that are deemed to be nonrepresentative (Guideline 5). Furthermore, a manual validation by domain experts (Guideline 6), where feasible, would further serve to ensure the precision of the extracted terms and could also be used to assign additional semantic categories such as sentiment.

Summary of guidelines is as follows:

1. Define the notion of sentiment for the clinical domain

2. Use corpus word frequencies as a guide to inclusion of words in a lexicon

3. Label terms as risk factor or trigger rather than sentiment-bearing

4. Use specialized dictionaries and/or health care terminologies as a source

5. Incorporate domain-specific corpus-based notion of representativeness into automatic lexicon induction techniques

6. Manual validation by domain experts

\section{Summary and Limitations}

Examining our data using the methods of corpus linguistics revealed statistically significant differences between the keywords used in EHR notes preceding an admission for attempted suicide and those from control periods not associated with such an attempt. Themes included hospitalized suicide attempts, self-harm, and alcohol. Coverage of these keywords by the general-purpose sentiment lexicons we reviewed was varied. Although lexicon size was a determining factor in overall coverage, the largest resource, SentiWordNet, did not distinguish the 2 subcorpora as well as some of the smaller resources, namely, AFINN, EmoLex, and Opinion, once both keyword rankings and sentiment were taken into consideration. Similarly, EmoLex and AFINN had wider coverage of relevant keywords than Opinion, which is the largest of the 3 resources. This may be partly a consequence of the original sampling strategy used to select words to construct sentiment lexicons. Both EmoLex 
and AFINN were built on top of existing general-purpose dictionaries, whereas Opinion was created semiautomatically by crawling product reviews on the internet. As a result, the vocabulary of the latter may be more specific to that domain, whereas the 2 former lexicons are likely to be more generic in their terminology, meaning they may adapt slightly better to different domains. The same 3 lexicons also showed the most discriminating assignment of sentiment polarity between the case and control keywords. Although many of the terms contained in these resources can be said to convey appropriate sentiment values (eg, anxiety is negative and pleasant is positive), there are also certain terms for which this is less obvious, at least in the context of EHR text related to suicide risk. For example, ward is assigned negative sentiment by SentiWordNet, whereas thoughts are assigned positive sentiment. The word plan is assigned positive sentiment by EmoLex, whereas call is negative. Annotating word polarity in a noncontextual manner, especially without appropriate part-of-speech disambiguation (only 2 of the resources we tested contained entries with part-of-speech information), could lead to biased analyses in downstream modeling of new use cases. Clinical texts are intended to be written in an objective style, rather lacking what one might generally term sentiment, although in reality this may not always be the case. Many of the most highly relevant terms identified by our approach (eg, $a \& e$, overdoses, and alcohol) do not fall into what might typically be termed a sentiment category but rather belong to categories of risk factors, whereas other identified terms are more sentiment bearing.

These observations lead us to concur with the conclusions of previous research [21-24] that domain-specific resources need to be developed for the analysis of clinical texts. We have attempted to provide insight into why this might be and what information such resources might need to include to address the task of suicide risk assessment through the analysis of clinical notes.

Our study has some limitations. First, the corpus was not constructed according to a deliberate sampling strategy but is the result of a 1:4 case-control selection ratio, which is typical in epidemiology. Completed and attempted suicide is much rarer than our sample suggests. Furthermore, the documents were not sampled according to type. This may have led to a preponderance of letters in the control corpus, as suggested by the most frequent keywords. The distribution of documents between patients also differs between the case and control subcorpora. Cases have, on average, almost 3 times the number of documents as controls, which is reflective of more frequent contact with mental health services. Consequently, the resulting corpus does not necessarily fulfill the criteria of representativeness and balance generally recommended in corpus linguistics.
We also acknowledge that our normalization of sentiment values for the sake of comparison does not necessarily reflect the actual quantity of sentiment assigned by all lexicons and invite the reader to refer to previous studies where raw sentiment scores are compared [20-22]. It is also worth noting that previous studies have shown that emotions, such as happiness expressed in social media posts, may vary with population demographics, geographical location [51,52], movement, and residency status in an area [53]. Although our work has focused on clinical texts instead of social media, such factors may have influenced our results; however, we have not controlled for this. This represents a caveat concerning the generalizability of our results to clinical populations in other geographical areas with potentially different sociodemographic configurations.

Finally, we only examined keywords that were common to both subcorpora. As a consequence, certain keywords typical of suicidal case notes only appearing in the case subcorpus may have been missed out, although we did find keywords appearing in only 1 subcorpus to be relatively infrequent compared with those we did examine.

\section{Conclusions}

This work makes several contributions to the study of sentiment in suicide risk assessment.

First, our corpus of clinical notes drawn from a case-control study of suicidal and nonsuicidal hospital admissions is, to our knowledge, a novel use of EHRs in this area.

Second, by applying methods of corpus linguistics, we identified 2 lists of keywords: the first representative of the clinical notes of patients leading up to a hospitalized suicide attempt and a second for those who made no such attempt. We used these lists of keywords to gauge the coverage of 6 sentiment lexicons over our corpus, using a number of measures, including information retrieval metrics, which we adapted for the purposes of our evaluation. Our study provided a novel examination of the content of these lexicons and their implications in relation to sentiment analysis as well as deeper insights into the characteristics of terms that distinguish suicide risk cases from controls in EHR text. Furthermore, we found that these general-domain resources assign polarity values that are sometimes not clinically meaningful or consistent with clinical judgments.

Finally, based on the outcomes of our study, we have suggested a set of simple and clear guidelines to facilitate the creation of more useful lexical resources for those seeking to assess risk of suicide through the analysis of clinical notes. Such targeted lexicons have the potential to advance research into the use of EHRs for the study of suicide risk in clinical populations by providing discriminative features for use in both rule-based and machine learning classification systems.

\section{Acknowledgments}

The authors wish to thank Jefrey Lijffijt and Paul Rayson for their advice on corpus linguistics and James Pennebaker for permission to use the LIWC lexicon. Any errors are the authors' own. RD is funded by a Clinician Scientist Fellowship (project e-HOST-IT) from the Health Foundation in partnership with the Academy of Medical Sciences, which also funds AB. This work was also partly supported by Health Data Research UK, an initiative funded by UK Research and Innovation, Department of Health and 
Social Care (England), and the devolved administrations, leading medical research charities, and the Maudsley Charity. This paper represents independent research partly funded (AR, RD, SV, and AB) by the National Institute for Health Research (NIHR) Biomedical Research Centre at South London and Maudsley NHS Foundation Trust and King's College London. The views expressed are those of the authors and not necessarily those of the NHS, the NIHR, or the Department of Health and Social Care. The funders had no role in the study design, data collection and analysis, decision to publish, or preparation of the manuscript.

\section{Authors' Contributions}

AB conceptualized the data curation, formal analysis, investigation, methodology, software, and writing-original draft preparation. SV conceptualized the methodology, and writing-review and editing. AR conceptualized and contributed in writing review and editing. RD conceptualized formal analysis, funding acquisition, supervision, writing-original draft preparation, and writing-review and editing.

\section{Conflicts of Interest}

$\mathrm{RD}$ and SV declare previous research funding received from Janssen.

\section{Multimedia Appendix 1}

Technical details of data and analyses.

[DOCX File, 102 KB-Multimedia Appendix 1]

\section{Multimedia Appendix 2}

Top 100 case and control keywords and associated polarities per lexicon.

[XLSX File (Microsoft Excel File), 31 KB-Multimedia Appendix 2]

\section{References}

1. Suicide data. World Health Organization. 2016. URL: http://www.who.int/mental health/prevention/suicide/suicideprevent/ en/ [accessed 2019-04-16]

2. Bostwick JM, Pabbati C, Geske JR, McKean AJ. Suicide Attempt as a Risk Factor for Completed Suicide: Even More Lethal Than We Knew. Am J Psychiatry 2016 Nov 01;173(11):1094-1100 [FREE Full text] [doi:

10.1176/appi.ajp.2016.15070854] [Medline: 27523496]

3. Franklin JC, Ribeiro JD, Fox KR, Bentley KH, Kleiman EM, Huang X, et al. Risk factors for suicidal thoughts and behaviors: A meta-analysis of 50 years of research. Psychol Bull 2017 Feb;143(2):187-232. [doi: 10.1037/bul0000084] [Medline: 27841450]

4. Velupillai S, Hadlaczky G, Baca-Garcia E, Gorrell GM, Werbeloff N, Nguyen D, et al. Risk assessment tools and data-driven approaches for predicting and preventing suicidal behavior. Front Psychiatry 2019 Feb 13;10:36 [FREE Full text] [doi: 10.3389/fpsyt.2019.00036] [Medline: 30814958]

5. Strauss J. Subjectivity and severe psychiatric disorders. Schizophr Bull 2011 Jan 20;37(1):8-13 [FREE Full text] [doi: 10.1093/schbul/sbq116] [Medline: 20961994]

6. Pang B, Lee L. Opinion mining and sentiment analysis. FNT in Information Retrieval 2008;2(1-2):1-135. [doi: 10.1561/1500000011]

7. Liu B. Sentiment Analysis: Mining Opinions, Sentiments, and Emotions. Cambridge, UK: Cambridge University Press; 2015.

8. Turney PD. Thumbs up or thumbs down?: semantic orientation applied to unsupervised classification of reviews. In: Proceedings of the 40th Annual Meeting on Association for Computational Linguistics - ACL'02.: Proceedings of the 40th Annual Meeting on Association for Computational Linguistics - ACL ?02. Association for Computational Linguistics; 2001 Presented at: The 40th Annual Meeting on Association for Computational Linguistics - ACL'02; July 2002; Philadelphia, PA, USA p. 417-424. [doi: 10.3115/1073083.1073153]

9. Hu M, Liu B. Mining and Summarizing Customer Reviews. In: Proceedings of the Tenth ACM SIGKDD International Conference on Knowledge Discovery and Data Mining. New York, NY, United States: Association for Computing Machinery; 2004 Presented at: The Tenth ACM SIGKDD International Conference on Knowledge Discovery and Data Mining; August 2004; Seattle, WA, USA p. 168-177 URL: https://doi.org/10.1145/1014052.1014073 [doi: 10.1145/1014052.1014073]

10. Blair-Goldensohn S, Hannan K, McDonald R. Building a Sentiment Summarizer for Local Service Reviews. In: Proceedings of NLP in the Information Explosion Era (NLPIX 2008). 2008 Presented at: NLP in the Information Explosion Era (NLPIX 2008); April 2008; Beijing, China URL: https://static.googleusercontent.com/media/research.google.com/en/ /pubs/archive/34368.pdf

11. Socher R, Perelygin A, Wu J. Recursive Deep Models for Semantic Compositionality Over a Sentiment Treebank. : Association for Computational Linguistics; 2013 Presented at: Conference on Empirical Methods in Natural Language Processing. Published online ?1642; 2013; Seattle, WA, USA p. 1631-1642. 
12. Pak A, Paroubek P. Twitter as a Corpus for Sentiment Analysis and Opinion Mining. In: Proceedings of LREC 2010. Luxembourg: European Language Resources Association (ELRA); 2010 Presented at: Language Resources and Evaluation Conference (LREC 2010); May 2010; Valleta, Malta p. 1320-1326.

13. Agarwal A, Xie B, Vovsha I, Rambow O, Passonneau R. Sentiment Analysis of Twitter Data. In: Proceedings of the Workshop on Languages in Social Media. Stroudsburg, PA, USA: Association for Computational Linguistics; 2011 Presented at: Workshop on Languages in Social Media (LSM'11); June 2011; Portland, OR, USA p. 30-38.

14. Dini L, Bittar A. Emotion Analysis on Twitter: the Hidden Challenge. In: Proceedings of LREC 2016. Luxembourg: European Language Resources Association (ELRA); 2016 Presented at: Language Resources and Evaluation Conference (LREC 2016); May 2016; Reykjavik, Iceland p. 3953-3958.

15. Smith P, Lee M. Cross-discourse Development of Supervised Sentiment Analysis in the Clinical Domain. Stroudsburg, PA, USA: Proceedings of the 3rd Workshop in Computational Approaches to Subjectivity and Sentiment Analysis. Association for Computational Linguistics; 2012 Presented at: Third Workshop in Computational Approaches to Subjectivity and Sentiment Analysis; July 2012; Jeju, Korea p. 79-83.

16. Xia C, Zhao D, Wang J, Liu J, Ma J. ICSH 2018: LSTM based Sentiment Analysis for Patient Experience Narratives in E-survey Tools. In: Chen H, Fang Q, Zeng D, Wu J, editors. Proceedings of ICSH 2018. Berlin/Heidelberg, Germany: Springer International Publishing; 2018:231-239.

17. Greaves F, Ramirez-Cano D, Millett C, Darzi A, Donaldson L. Use of sentiment analysis for capturing patient experience from free-text comments posted online. Journal of Medical Internet Research 2013;15(11):e239 [FEE Full text] [doi: 10.2196/jmir.2721] [Medline: 24184993]

18. Wang X, Zhang C, Ji Y, Sun L, Wu L, Bao Z. A Depression Detection Model Based on Sentiment Analysis in Micro-blog Social Network. In: Li J, Cao L, Wang C, Tan KC, Liu B, Pei J, et al, editors. Trends and Applications in Knowledge Discovery and Data Mining. Berlin/Heidelberg, Germany: Springer; 2013:201-213.

19. Tao X, Zhou X, Zhang J, Yong J. Sentiment Analysis for Depression Detection on Social Networks. In: Li J, Li X, Wang S, Li J, Sheng QZ, editors. Advanced Data Mining and Applications. Berlin/Heidelberg, Germany: Springer International Publishing; 2016:807-810.

20. McCoy TH, Castro VM, Cagan A, Roberson AM, Kohane IS, Perlis RH. Sentiment Measured in Hospital Discharge Notes Is Associated with Readmission and Mortality Risk: An Electronic Health Record Study. PLoS ONE 2015 Aug 24;10(8):e0136341. [doi: 10.1371/journal.pone.0136341]

21. McCoy TH, Castro VM, Roberson AM, Snapper LA, Perlis RH. Improving Prediction of Suicide and Accidental Death After Discharge From General Hospitals With Natural Language Processing. JAMA Psychiatry 2016 Oct 1;73(10):1064-1071. [doi: 10.1001/jamapsychiatry.2016.2172] [Medline: 27626235]

22. Weissman GE, Ungar LH, Harhay MO, Courtright KR, Halpern SD. Construct validity of six sentiment analysis methods in the text of encounter notes of patients with critical illness. J Biomed Inform 2019 Jan;89:114-121 [FREE Full text] [doi: 10.1016/j.jbi.2018.12.001] [Medline: 30557683]

23. Deng Y, Stoehr M, Denecke K. Retrieving Attitudes: Sentiment Analysis from Clinical Narratives. In: Proceedings of the Medical Information Retrieval (MedIR) Workshop. New York, NY, USA: Association for Computing Machinery; 2014 Jul 11 Presented at: 37th international ACM SIGIR conference on Research \& development in information retrieval; July 2014; Gold Coast, Australia p. 12-15.

24. Deng Y, Declerck T, Lendvai P, Denecke K. The Generation of a Corpus for Clinical Sentiment Analysis. In: Sack H, Rizzo G, Steinmetz N, Mladenić D, Auer S, Lange C, editors. Lecture Notes in Computer Science. Cham, Switzerland: Springer International Publishing; 2016:311-324.

25. Holderness E, Cawkwell P, Bolton K, Pustejovsky J, Hall MH. Distinguishing Clinical Sentiment: The Importance of Domain Adaptation in Psychiatric Patient Health Records. In: Proceedings of the 2nd Clinical Natural Language Processing Workshop. Stroudsburg, PA, United States: Association for Computational Linguistics; 2019 Presented at: 2nd Clinical Natural Language Processing Workshop; June 2019; Minneapolis, MN, USA p. 117-123. [doi: 10.18653/v1/w19-1915]

26. Perera G, Broadbent M, Callard F, Chang C, Downs J, Dutta R, et al. Cohort profile of the South London and Maudsley NHS Foundation Trust Biomedical Research Centre (SLaM BRC) Case Register: current status and recent enhancement of an Electronic Mental Health Record-derived data resource. BMJ Open 2016 Mar 1;6(3):e008721 [FREE Full text] [doi: 10.1136/bmjopen-2015-008721] [Medline: 26932138]

27. Grimes DA, Schulz KF. Compared to what? Finding controls for case-control studies. Lancet 2005 Apr;365(9468):1429-1433. [doi: 10.1016/s0140-6736(05)66379-9]

28. spaCy - Industrial-strength Natural Language Processing in Python. URL: https://spacy.io/ [accessed 2020-07-07]

29. Bird S, Klein E, Loper E, Baldridge J. Multidisciplinary instruction with the Natural Language Toolkit. In: Proceedings of the Third Workshop on Issues in Teaching Computational Linguistics (TeachCL'08). Stroudsburg, PA, USA: Association for Computational Linguistics; 2008 Presented at: Third Workshop on Issues in Teaching Computational Linguistics (TeachCL'08); June 2008; Columbus, OH, USA p. 62-70. [doi: 10.3115/1627306.1627317]

30. KCL-Health-NLP/Suicide-Risk-Sentiment. URL: https://github.com/KCL-Health-NLP/suicide-risk-sentiment [accessed 2020-11-09]

31. Kilgarriff A. Comparing Corpora. IJCL 2001 Dec 17;6(1):97-133. [doi: 10.1075/ijcl.6.1.05kil] 
32. Paquot M, Bestgen Y. Distinctive words in academic writing: A comparison of three statistical tests for keyword extraction. In: Jucker AH, Schreier D, Hundt M, editors. Pragmatics and Discourse. Leiden, Netherlands: Brill; 2009.

33. Lijffijt J, Nevalainen T, Säily T, Papapetrou P, Puolamäki K, Mannila H. Significance testing of word frequencies in corpora. Digital Scholarship Humanities 2014 Dec 8;31(2):374-397. [doi: 10.1093/llc/fqu064]

34. Gries ST. Dispersions and adjusted frequencies in corpora. IJCL 2008;13(4):403-437. [doi: 10.1075/ijcl.13.4.02gri]

35. Nielsen FÅ. A new ANEW: Evaluation of a word list for sentiment analysis in microblogs.: arXiv; 2011. URL: http://arxiv. org/abs/1103.2903 [accessed 2019-02-25]

36. Mohammad S, Turney P. Crowdsourcing a Word-Emotion Association Lexicon. Computational Intelligence 2013;29(3):436-465. [doi: 10.1111/j.1467-8640.2012.00460.x]

37. Tausczik YR, Pennebaker JW. The Psychological Meaning of Words: LIWC and Computerized Text Analysis Methods. Journal of Language and Social Psychology 2009 Dec 08;29(1):24-54. [doi: 10.1177/0261927X09351676]

38. De Smedt T, Daelemans W. Pattern for Python. J Mach Learn Res 2012;13:2063-2067.

39. Baccianella S, Esuli A, Sebastiani F. SENTIWORDNET 3.0: An Enhanced Lexical Resource for Sentiment Analysis and Opinion Mining. In: Proceedings of the 7th edition of the Language Resources and Evaluation Conference (LREC 2010). 2010 Presented at: Language Resources and Evaluation Conference (LREC 2010); 2010; Valleta, Malta p. A.

40. Miller GA. WordNet: a lexical database for English. Commun ACM 2019;38(11):39-41. [doi: 10.1145/219717.219748]

41. Bernard J. The Macquarie Thesaurus. In: Macquarie Library. Sydney, Australia: Macquarie Library; 1986.

42. Strapparava C, Valitutti A. WordNet-Affect: an Affective Extension of WordNet. In: Proceedings of the Fourth International Conference on Language Resources and Evaluation (LREC 2004). 2004 Presented at: Language Resources and Evaluation Conference (LREC 2004); May 2004; Lisbon, POrtugal.

43. Stone P, Dunphy D, Smith M, Ogilvie D. The General Inquirer: A Computer Approach to Content Analysis. Cambridge, MA, USA: MIT Press; 1966.

44. Brants T, Franz A. Web 1T 5-Gram Version 1.: Linguistic Data Consortium; 2006. URL: http://www.ldc.upenn.edu/Catalog/ CatalogEntry.jsp?catalogId=LDC2006T13 [accessed 2019-04-20]

45. Fernandes AC, Cloete D, Broadbent MT, Hayes RD, Chang C, Jackson RG, et al. Development and evaluation of a de-identification procedure for a case register sourced from mental health electronic records. BMC Med Inform Decis Mak 2013 Jul 11;13:71 [FREE Full text] [doi: 10.1186/1472-6947-13-71] [Medline: 23842533]

46. Hettne KM, van Mulligen EM, Schuemie MJ, Schijvenaars BJ, Kors JA. Rewriting and suppressing UMLS terms for improved biomedical term identification. J Biomed Semantics 2010 Mar 31;1(1):5 [FREE Full text] [doi: 10.1186/2041-1480-1-5] [Medline: 20618981]

47. Tai YJ, Kao HY. Automatic Domain-Specific Sentiment Lexicon Generation with Label Propagation. In: Proceedings of International Conference on Information Integration and Web-Based Applications \& Services (IIWAS'13). New York, NY, USA: Association for Computing Machinery; 2013 Presented at: International Conference on Information Integration and Web-Based Applications \& Services (IIWAS'13); December 2013; Vienna, Austria p. 53-62. [doi: 10.1145/2539150.2539190]

48. Hamilton W, Clark K, Leskovec J, Jurafsky D. Inducing Domain-Specific Sentiment Lexicons from Unlabeled Corpora.: arXiv URL: http://arxiv.org/abs/1606.02820 [accessed 2019-04-19]

49. Yang X, Zhang Z, Zhang Z, Mo Y, Li L, Yu L, et al. Automatic construction and global optimization of a multisentiment lexicon. Comput Intell Neurosci 2016;2016:2093406-2093408 [FREE Full text] [doi: 10.1155/2016/2093406] [Medline: $\underline{28042290]}$

50. Pryzant R, Shen K, Jurafsky D, Wagner S. Deconfounded Lexicon Induction for Interpretable Social Science. In: Proceedings of the 2018 Conference of the North American Chapter of the Association for Computational Linguistics: Human Language Technologies, Volume 1 (Long Papers). Stroudsburg, PA, USA: Association for Computational Linguistics; 2018 Presented at: Human Language Technologies; June 2018; New Orleans, LA, USA p. 1615-1625. [doi: 10.18653/v1/n18-1146]

51. Mitchell L, Frank MR, Harris KD, Dodds PS, Danforth CM. The geography of happiness: connecting twitter sentiment and expression, demographics, and objective characteristics of place. PLoS One 2013 May 29;8(5):e64417 [FREE Full text] [doi: 10.1371/journal.pone.0064417] [Medline: 23734200]

52. Gore RJ, Diallo S, Padilla J. You are what you tweet: connecting the geographic variation in America's obesity rate to twitter content. PLoS One 2015;10(9):e0133505 [FREE Full text] [doi: 10.1371/journal.pone.0133505] [Medline: 26332588]

53. Padilla JJ, Kavak H, Lynch CJ, Gore RJ, Diallo SY. Temporal and spatiotemporal investigation of tourist attraction visit sentiment on Twitter. PLoS One 2018 Jun 14;13(6):e0198857 [FREE Full text] [doi: 10.1371/journal.pone.0198857] [Medline: 29902270]

\section{Abbreviations}

CRIS: Clinical Record Interactive Search

EHR: electronic health record

Freq: frequency

FreqDiff: frequency difference

FreqRatio: frequency ratio 
ICD: International Classification of Diseases

LIWC: Linguistic Inquiry and Word Count

NLP: natural language processing

Edited by C Lovis; submitted 10.07.20; peer-reviewed by S Mohammad, $R$ Gore; comments to author 20.09.20; revised version received 26.11.20; accepted 05.12.20; published 13.04.21

Please cite as:

Bittar A, Velupillai S, Roberts A, Dutta R

Using General-purpose Sentiment Lexicons for Suicide Risk Assessment in Electronic Health Records: Corpus-Based Analysis

JMIR Med Inform 2021;9(4):e22397

URL: https://medinform.jmir.org/2021/4/e22397

doi: 10.2196/22397

PMID:

(C)André Bittar, Sumithra Velupillai, Angus Roberts, Rina Dutta. Originally published in JMIR Medical Informatics (http://medinform.jmir.org), 13.04.2021. This is an open-access article distributed under the terms of the Creative Commons Attribution License (https://creativecommons.org/licenses/by/4.0/), which permits unrestricted use, distribution, and reproduction in any medium, provided the original work, first published in JMIR Medical Informatics, is properly cited. The complete bibliographic information, a link to the original publication on http://medinform.jmir.org/, as well as this copyright and license information must be included. 